\title{
antibacTR: dynamic antibacterial-drug-target ranking integrating comparative genomics, structural analysis and experimental annotation
}

\author{
Alejandro Panjkovich ${ }^{1}$, Isidre Gibert ${ }^{1,2}$ and Xavier Daura ${ }^{1,3^{*}}$
}

\begin{abstract}
Background: Development of novel antibacterial drugs is both an urgent healthcare necessity and a partially neglected field. The last decades have seen a substantial decrease in the discovery of novel antibiotics, which combined with the recent thrive of multi-drug-resistant pathogens have generated a scenario of general concern. The procedures involved in the discovery and development of novel antibiotics are economically challenging, time consuming and lack any warranty of success. Furthermore, the return-on-investment for an antibacterial drug is usually marginal when compared to other therapeutics, which in part explains the decrease of private investment.

Results: In this work we present antibacTR, a computational pipeline designed to aid researchers in the selection of potential drug targets, one of the initial steps in antibacterial-drug discovery. The approach was designed and implemented as part of two publicly funded initiatives aimed at discovering novel antibacterial targets, mechanisms and drugs for a priority list of Gram-negative pathogens: Acinetobacter baumannii, Escherichia coli, Helicobacter pylori, Pseudomonas aeruginosa and Stenotrophomonas maltophilia. However, at present this list has been extended to cover a total of 74 fully sequenced Gram-negative pathogens. antibacTR is based on sequence comparisons and queries to multiple databases (e.g. gene essentiality, virulence factors) to rank proteins according to their potential as antibacterial targets. The dynamic ranking of potential drug targets can easily be executed, customized and accessed by the user through a web interface which also integrates computational analyses performed in-house and visualizable on-site. These include three-dimensional modeling of protein structures and prediction of active sites among other functionally relevant ligand-binding sites.
\end{abstract}

Conclusions: Given its versatility and ease-of-use at integrating both experimental annotation and computational analyses, antibacTR may effectively assist microbiologists, medicinal-chemists and other researchers working in the field of antibacterial drug-discovery. The public web-interface for antibacTR is available at 'http://bioinf.uab.cat/ antibactr'.

Keywords: Antibacterial-drug target, Gram-negative bacteria, Target druggability

\footnotetext{
${ }^{*}$ Correspondence: xavier.daura@uab.cat

1 Institute of Biotechnology and Biomedicine (IBB), Universitat Autònoma de

Barcelona (UAB), 08193 Bellaterra, Spain

${ }^{3}$ Catalan Institution for Research and Advanced Studies (ICREA), 08010

Barcelona, Spain

Full list of author information is available at the end of the article
} 


\section{Background}

Since their initial discovery and application during the early 20th century, antibiotics have been playing a key role in public health worldwide. These 'miracle drugs' have contributed significantly to the increase in life expectancy since the end of World War II. Besides curing infections, they also prevent amputations and blindness and are involved in multiple healthcare procedures such as joint-replacement, surgery, new cancer treatments, etc [1]. However, after peaking during the 1960's, the discovery of new antibiotics has fallen off dramatically. The present scarcity of novel antibiotics becomes a major health concern in light of the remarkable ability of bacteria to rapidly evolve resistance mechanisms which erode the therapeutic effect of known antibiotics [2]. Nowadays multi-drug-resistant bacterial infections are increasing in both developing and developed countries and in both community and nosocomial settings [3]. It has been reported that a number of pathogens, including Staphylococcus aureus, Mycobacterium tuberculosis, Pseudomonas aeruginosa, Acinetobacter baumannii and some Enterobacteriaceae have developed resistance to a wide range of antimicrobial agents at an alarming rise, with some strains becoming truly pan-resistant $[4,5]$. However, pharmaceutical companies have not been investing in the development of new antibacterial drugs with corresponding efforts, mainly due to economic criteria that favour other therapeutic areas with better return-on-investment ratios $[6,7]$. The few antibacterial agents that have been launched during the last decade (e.g. linezolid, daptomycin) have a good activity against Gram-positive bacteria such as methicillin-resistant $S$. aureus (MRSA) and vancomycin-resistant enterococci [8]. However, cases of resistance for these new Gram-positive antibiotics have been reported recently as well [9].

The situation is worse for Gram-negative bacteria, such as $P$. aeruginosa and $A$. baumannii, which are common among nosocomial infections [10] and for which no new antibiotics have reached advanced stages of development [1]. In addition, with the increase in the prevalence of extended spectrum $\beta$-lactamase (ESBL)-producing Enterobacteriaceae, the use of carbapenems, a potential alternative to treat infections caused by these microorganisms, is leading to the emergence of multi-drug-resistant Enterobacteriaceae including resistance to carbapenems [11].

This scenario emphasizes the relevance of initiatives focused on the discovery of novel targets and antibacterials for combating Gram-negative pathogens. Here, we describe a tool (antibacTR: antibacterial Target Ranking) to support the initial stages of selection of potential antibacterial-drug targets, developed within the context of two such initiatives. antibacTR integrates a database with a pipeline that ranks and filters proteins according to a set of criteria commonly associated to antibacterial targets.
The approach is based on protein sequence comparisons, for which we developed an unbiased measure described in the Methods Section.

The interface used to interrogate the database and access the results has the form of a web-based tool, which has been developed following the suggestions of the experimentalists involved in the two target-discovery initiatives. It includes access to thousands of threedimensional protein-structure models that can be visualized and downloaded for further analysis through the web-interface. To further exploit the structural models, we incorporated a predictive approach that evaluates putative ligand-binding pockets in terms of their potential to affect protein function upon ligand binding $[12,13]$. Links to DrugBank [14] and the Virulence Factors DataBase (VFDB) [15], as well as predictions of active-site residues are provided as well. It should be noted that the final aim of the tool is both to rank proteins according to the chosen set of criteria (with weights defined by the user) and to provide for each protein in the ranked list information that could be relevant to antibacterial-drug-target selection. Clearly, the drug-target property is the result of a complex combination of a variable number of nonuniversal factors, some of which having opposite sign for different types of targets or mechanisms. Thus, this is a tool to support target discovery efforts, not a targetprediction tool. In other words, it will not spare the user from scanning and evaluating a large number of proteins, it will simply provide him/her with additional means to do it.

All measurements and predictions are pre-computed, which allows the application to return full rankings and links to the relevant information within seconds. This characteristic distinguishes our tool from similar ones such as the UniDrug-Target (UDT) database, which can be used to perform comparative analyses online with computation at time of request [16]. Besides execution speed, our approach differs from UDT and related ones such as the Prokaryotic-genome Analysis Tool (PGAT) [17] in its focus. While these tools succeed at providing comparative-analysis means that can be used through a web-interface, our dynamic approach focuses on speed, ease of use and an integrative solution that allows the user to quickly scan putative antibacterial targets and relevant information such as three-dimensional structural models and other predictions, while the comparative analysis is just one of the underlying features.

Other researchers have focused on extensive, manually curated analyses of a single organism and strain, like Shanmugham and Pan [18] on Mycobacterium abscessus ATCC 19977. Instead, we have chosen to sacrifice part of the depth of our analysis to cover a much larger selection of pathogens. The methodology presented here was originally developed for two specific projects and their 
target bacteria, including three of the big four Gramnegative pathogens in relation to systemic infections, i.e. Escherichia coli, Klebsiella pneumoniae, Acinetobacter baumannii and Pseudomonas aeruginosa [4]. However, we have now expanded the coverage to include 74 Gramnegative pathogens, which should make the system useful to many more researchers in the field. Through this article, we describe the approach and make the web-based tool publicly available.

\section{Results}

\section{Computational target-ranking pipeline}

Typically, one of the first steps in a target-discovery project is to readily select, among thousands of proteins composing the pathogens' proteomes, those with the highest chance of becoming useful therapeutic targets. Following the lines defined by previous studies [19], we developed an algorithm to score and rank potential drug targets in pathogenic organisms by evaluating a modular set of criteria that are commonplace in antimicrobialdevelopment efforts [7]: 1) the presence of the protein in different pathogens, 2) evolutionary conservation, 3) essentiality, 4) presence of isoforms and paralogs in the proteome, 5) similarity to human proteins. We implemented a set of five weighted scores that cover these criteria and defined a scoring function combining them.

The first two concepts were incorporated as two independent scores, measuring the conservation of the protein among Gram-negative organisms and among different strains of the same species, respectively. Conservation among strains is a basic requirement for target consideration. Conservation among Gram-negative species is highly desirable as it enables the development of broad-spectrum solutions and increases economic viability. In addition, well conserved targets will presumably have low tolerance to mutations, decreasing the chance of resistance to emerge by this type of mechanism.

Essential proteins, which inhibition compromises bacterial viability, are potential antibacterial targets by definition. We implemented a binary score by marking genes known to be essential from previous experimental work [20].

The remaining two scores are given negative weights. If the protein under consideration has isoforms and/or paralogs the pathogen may readily develop resistance by functional substitution, and the effect of the antibacterial may be also reduced by competitive binding to non-essential forms. We considered similarity to human proteins negative as well, since close human homologs to the target may interact with the drug, giving rise to unwanted side-effects.

The scoring and ranking scheme, partially following the work of White and Kell [19] provides an advantage when compared to static selection or filtering approaches
$[16,21]$. In our case, if further experimental analysis reveals that a given protein is not suitable as a drug target, work can continue with the next protein in the ranking. Moreover, it would be straightforward to incorporate new criteria into the ranking scheme if needed.

The pipeline to which each proteome of interest was subjected is illustrated in Figure 1, which summarizes the approach.

\section{Sequence-based analysis}

Currently, the database covers 74 Gram-negative pathogens, including 224 distinct strains. In the case of Acinetobacter baumannii, Escherichia coli, Helicobacter pylori, Pseudomonas aeruginosa and Stenotrophomonas maltophilia, which are pathogens distinguished by their prevalence in community and/or nosocomial infections and the incidence of drug-resistant isolates [3,10], we included all available fully sequenced strains (82 strains, which conform a 'priority set'). As for the rest of the species, we included all strains that were marked as 'human pathogens' in HAMAP (142 strains) [22]. This query data set was compared against the human proteome and a reference set of $770 \mathrm{Gram}$-negative proteomes (494 distinct species), by means of the BLASTPGP program [23] using default parameters. BLASTPGP searches are very fast, however resulting E-values depend on the alignment itself and on other parameters such as the size of the database scanned. We needed unbiased similarity scores between proteins matched during the sequence-based search to keep results valid in case of further increasing the size of the data sets. To attain this objective, we further aligned BLASTPGP matches (E-value $<=0.0001$ ) using the Smith-Waterman algorithm and calculated 'normalized sequence similarity scores' (NS). NS values were then used to pre-compute toxicity, presence of isoforms or paralogs and the two conservation scores for each protein in the query data set, as described in further detail in the Methods Section.

\section{Queries to external databases}

Besides comparative analysis, a round of database queries was also performed to integrate additional information. Thus, protein sequences were compared to the list of known drug-targets available at the DrugBank database [14] and sequence-based searches were also performed against virulence factors available at the Virulence Factors DataBase (VFDB) [15]. Out of the total 777,585 proteins in the query data set, 375,016 matched a known target in DrugBank (48\%) and 193,135 proteins matched a known virulence factor at VFDB (25\%). This information was not incorporated as ranking scores, but it is available through the web-interface described below for researchers to evaluate themselves the relevance of such matches in each particular case. 


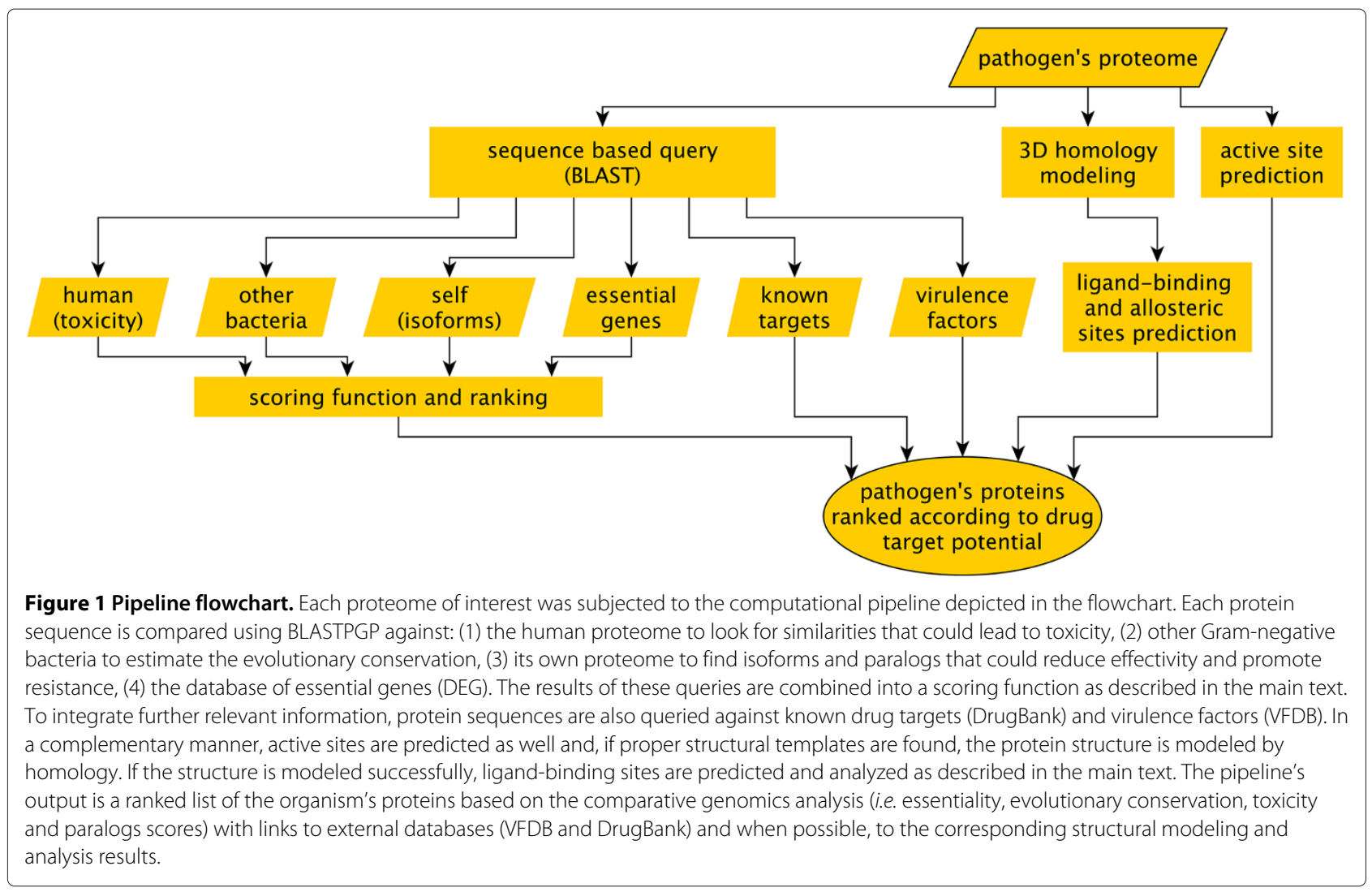

\section{Three-dimensional homology modeling}

Researchers evaluating prospective drug targets may benefit from the availability of protein structural data. For the organisms in the priority set, we performed a large-scale homology modeling of all protein sequences for which we found valid structural templates as explained in the Methods Section. In total, we generated threedimensional homology models for 136,141 proteins (covering $47 \%$ of the priority set). This number was obtained after discarding models presenting less than $30 \%$ sequence identity (target-template) or G-factors below -1.00 [24]. All models were generated by means of the MODELLER program [25] using default parameters.

To save computational power, proteins belonging to other strains were not modeled automatically. However, if the user is interested in obtaining one of such homology models, we have implemented an option at the webinterface for automatic submission of the selected modeling task.

\section{Active-site prediction}

To further add relevant information on putative targets, we applied a sequence-based approach [26] to predict the location of active-site residues. The method is based on comparing query sequences to homologs for which the position of the active site has been annotated. After analyzing the whole query set ( 777,585 proteins), this procedure predicted the location of active-site residues for $90,482$ proteins ( $11.6 \%)$.

Proteins with a predicted active site display a link to the details of the prediction in the web interface described below.

\section{Pocket analysis}

For proteins for which we could build a three-dimensional homology model, we predicted the location of ligandbinding sites on the structure using LIGSITEcs [27]. We further analyzed the ligand-binding sites using two previously developed methodologies which estimate the regulatory potential of particular ligand-binding pockets. When possible, the structural conservation of predicted pockets was measured considering the evolutionary record of the protein family, given that conserved pockets may have a relevant biological role [12]. Furthermore, using Normal Mode Analysis we estimated the effect of ligand binding on overall protein flexibility, a measure which has been used in combination with structural conservation to predict the location of allosteric sites [13]. As described below, the user can visualize the protein structure and predictions online. 


\section{Interface and access to results}

Interactive access to results is available through the webinterface at 'http://bioinf.uab.cat/antibactr'.

This interface allows the user to select the organisms and strain of interest, set custom weights to the different scores and then proceed to calculate the corresponding ranking. If the user wishes to ignore a specific ranking parameter, a weight of 0 (zero) can be applied. The system has been built in such a way that normalization of scores is performed only among the selected set of strains and parameters. To further facilitate the analysis of results, the user may also limit the amount of top-ranked entries that are displayed. Once the ranking procedure is finished (it takes a few seconds), the ranking is printed to the browser. An option is available for downloading the ranking to the local computer in tab-delimited text format, useful for researchers interested in further processing the data. Targets are displayed in ranked order and individual scores are shown for each protein after normalization but prior to weighting. A brief description of the biological function is displayed for each protein but, to facilitate immediate access to full annotation and other relevant data, a link to the related Uniprot entry is provided as well [28]. In cases where the target shows sequence similarity to an already known drug target or virulence factor, the corresponding links are also provided. In addition, specific links with details on predicted active sites and homology models are given. If a homology model is supplied, the user may download model coordinates in PDB format and target-template alignments generated during the modeling process, along with sequence identity, DOPE score and other relevant modeling data [25]. Furthermore, available protein structures can be visualized using Jmol (http://www.jmol.org) along with the results of the pocket analysis previously described [13].

\section{User query sequences}

Besides the ranking of complete proteomes, researchers may want to look at the ranking of a few selected proteins of their particular interest. To achieve this functionality, we added the possibility to include the user's own query sequences in an optional field. These sequences are then compared using BLASTPGP against our query data set (224 strains). Scoring and ranking proceed as normally, but results are then displayed only for significant hits within our data set. Details of this BLASTPGP search are also available to the user.

\section{Discussion}

Large-scale comparison of organisms at the genome level is a technique common to many fields of biology and medicine. In the past years complex approaches involving phylogenetic and metabolic studies have been published $[29,30]$. However, comparative genomics initiatives in drug discovery have been criticized for their limited success in finding new active compounds [31,32]. Yet, comparative genomics and proteomics continue to shed light on the workings of bacterial drug-resistance and virulence [33,34].

Far from attempting to solve the problem of target identification in one strike, our motivation was to implement a straightforward computational approach that would prove useful as an initial filtering and ranking step, aiding researchers in the quest for novel drug targets.

At the time of this writing no equivalent tool to the one presented here is available, however a few servers provide slightly related functionalities and could be used in a complementary fashion. For example the Prokaryotic-genome Analysis Tool (PGAT), developed by Brittnacher and collaborators is a general comparative genomics tool focused particularly on comparing different strains of the same species [17]. PGAT allows the user to carry out a series of interesting analyses including information on metabolic pathways, but it does not provide specific drug-target related information, unlike the UniDrug-Target (UDT) database which clearly focuses on that aspect [16]. The latter presents candidate targets as proteins which are present in pathogenic bacteria but absent in commensal strains. This is a reasonable approach which in our tool can be achieved by setting a negative value for the strain conservation score and it is also one of the functions available at PGAT. However, given its focus on pathogenspecific proteins UDT's approach tends to discard evolutionary conserved proteins, such as many well known broad-spectrum targets [35].

The amount of well known and characterized protein drug targets is currently in the order of hundreds [35]. To illustrate the potential of the tool presented here, we provide a few examples of already known antibacterial targets. Certain proteins involved in the replication of DNA are targeted by fluoroquinolones $[35,36]$. For example, Ciprofloxacin targets DNA topoisomerase 4 subunit B and DNA gyrase subunit A. Even though resistance to fluoroquinolones has been observed in pathogens with mutations in these proteins, new compounds with antibacterial activity on the resistant strains are being developed by studying these targets [37]. These proteins appear on the top $3 \%$ of the full proteome ranking for Escherichia coli K12 (positions 68 and 158, respectively) when we build the ranking using default parameters. This is because both proteins are essential, show high levels of evolutionary conservation but low similarity to human proteins (minimal potential toxicity) and present no isoforms or paralogs according to our pipeline parameters.

Beyond filtering and ranking targets, researchers can also gain insight into potential targets through the 
structural analysis methods we have implemented into the tool. For example, peptide deformylase [SwissProt:Q9I7A8] from Pseudomonas aeruginosa is an essential protein targeted by the antibiotic Actinonin [38]. This protein is ranked at position 81 among the complete Pseudomonas aeruginosa proteome (top 2\%) when using default parameters. Our pipeline automatically builds three-dimensional homology models when possible, it then predicts putative ligand-binding sites and evaluates their potential to regulate protein activity as described previously [13]. In this case, the structural analysis (which is pre-calculated and available through the web-interface) predicts one of the putative ligand-binding sites to significantly affect protein flexibility as shown in Figure 2. When we superimpose the automatically generated homology model with the known structure of the protein bound to the antibiotic ([PDB:1LRY] RMSD 0.5) the position of the cavity predicted to be significant matches precisely the location of the antibiotic molecule. This cavity is also considered relevant from an evolutionary perspective, as it shows $100 \%$ of structural conservation within its domain family according to the corresponding automatic analysis [12]. The structural conservation of this pocket was to be expected, since it is the protein's active site.

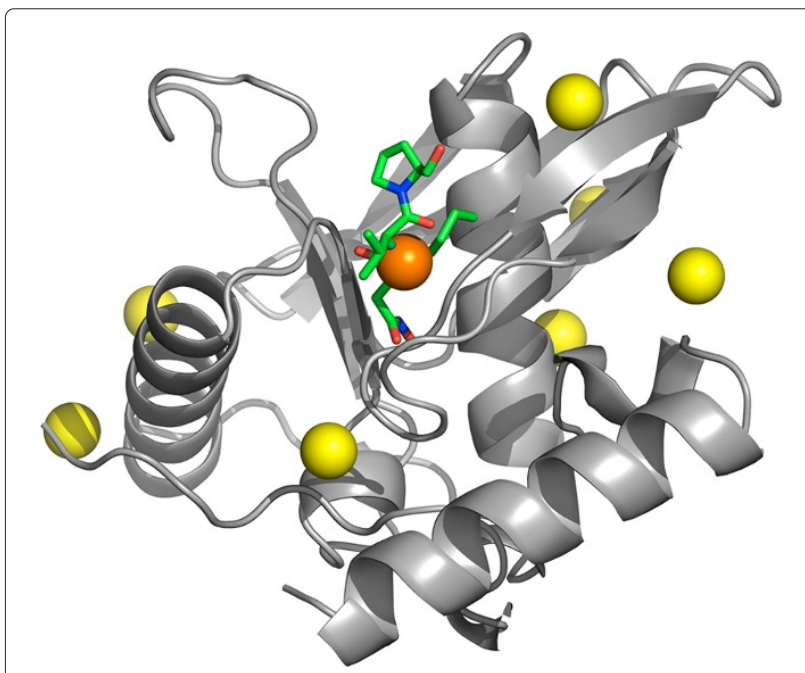

Figure 2 Peptide deformylase, automatic structural modeling and pocket analysis. The spheres displayed on the homology model of Pseudomonas aeruginosa peptide deformylase [Swiss-Prot:Q917A8] (based on template [PDB:1 N5N]) represent putative ligand-binding sites as predicted by the automatic pocket analysis. The orange sphere marks the only cavity predicted to significantly affect overall protein flexibility. To illustrate the relevance of this prediction, we show the location of the antibiotic ligand (in 'sticks' representation) after superimposing the homology model to the known structure of the antibiotic-bound protein [PDB:1LRY] (RMSD $0.5 \AA$ ). The position of antibiotic Actinonin matches precisely the cavity marked by the procedure. The same cavity is also estimated to be very well conserved at the structural level (100\% presence in the protein family).
Briefly, this case illustrates how in the situation of a poorly characterized protein, our automatic structural analysis may pinpoint not only the potential of the protein as a drug target, but the precise location of the drug-binding pocket.

Another interesting example is 3-oxoacyl-[acyl-carrierprotein] synthase III, which is involved in fatty-acid synthesis. This protein is targeted by Cerulenin, with antifungal effects, and it is a potential antibacterial target as well [39-41]. It is ranked by our pipeline at position 49 (top 2\%) among the full proteome of Escherichia coli K12 because it is essential and well conserved, showing in principle no toxicity (similarity to human proteins). Furthermore, structural analysis reveals one single pocket that could affect the protein's function by perturbing its overall flexibility, as shown in Figure 3. When we evaluate the structural prediction performed on the homology model by superimposing the known structure of the inhibitorbound protein, we observe that in this case the match of the cavity's geometric center is not as precise as in the previous example of peptide deformylase. Nevertheless, visual inspection shows that the large cavity indicated by the pocket structural analysis is indeed occupied by the

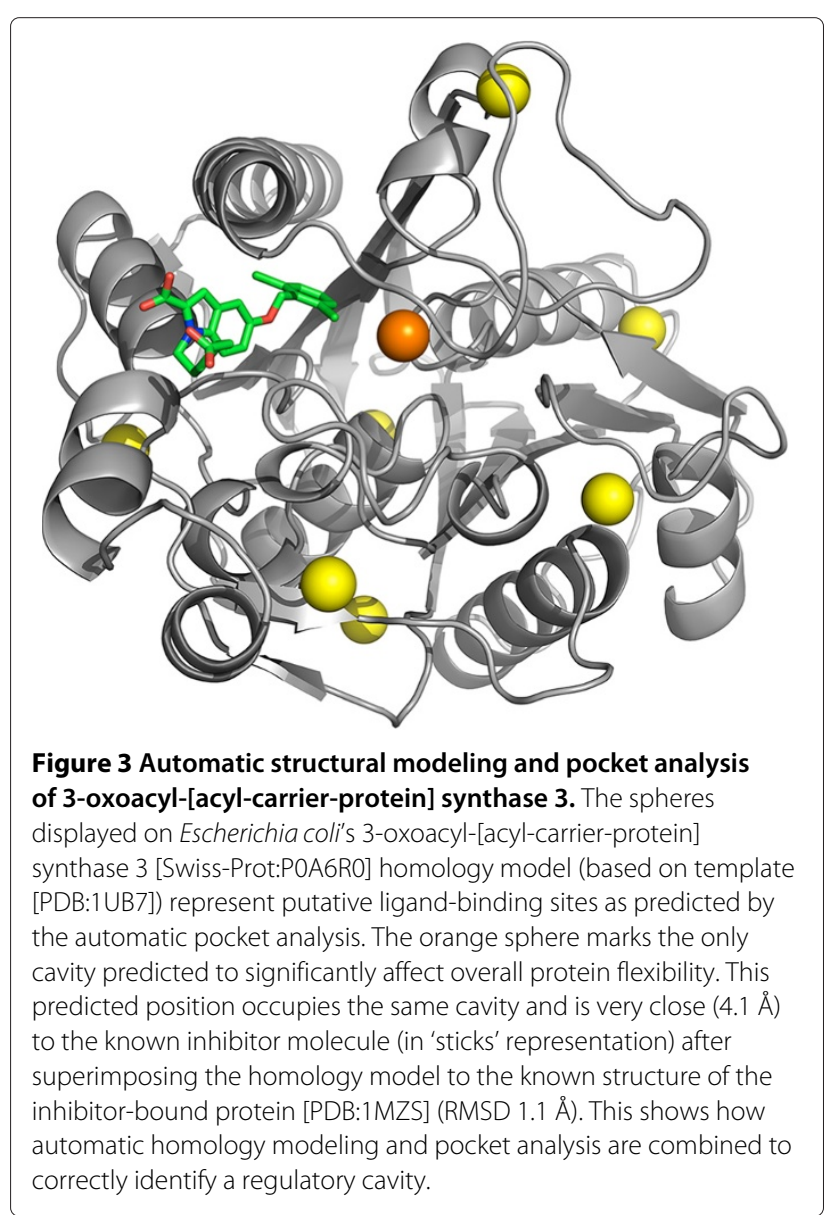


inhibitor, so that the automatic procedure would again be pointing the researcher in the right direction, even if no inhibition information would have been available a priori.

Of course, multiple other factors beyond the reach of a mere computational approach participate in defining a protein as a good antibacterial target. Indeed the final outcome of clinical trials can hardly be predicted [31]. However, we considered relevant to infer how well the pipeline presented in this work ranks already known targets. We gathered all known targets of 'approved' drugs, as annotated in DrugBank [14], that belonged to any of the strains analyzed in this work. We found a total of 57 proteins identified through their Uniprot ID [28]. Of those 57, a majority (48) belong to Escherichia coli $\mathrm{K} 12$. When we proceed to rank this organism's proteome, half of the known drug targets appear at the top $10 \%$ of the ranking. The distribution of the 48 known targets in the ranking of Escherichia coli $\mathrm{K} 12$ is displayed in Figure 4. It is interesting to note that the first half or top $10 \%$ correspond to essential proteins. Since essentiality is a binary score (i.e. genes may be essential or not), it divides the ranking in two sections as can be seen in the histogram (Figure 4). This illustrates the difficulty of a priori ranking antibacterial targets, since even though essentiality is considered a very desirable property for any candidate target $[32,42,43]$, it only represents half of the known targets in this organism. Moreover, assessing gene essentiality is not a trivial task, given that in vitro results do not always correlate with gene essentiality determined in vivo [43].

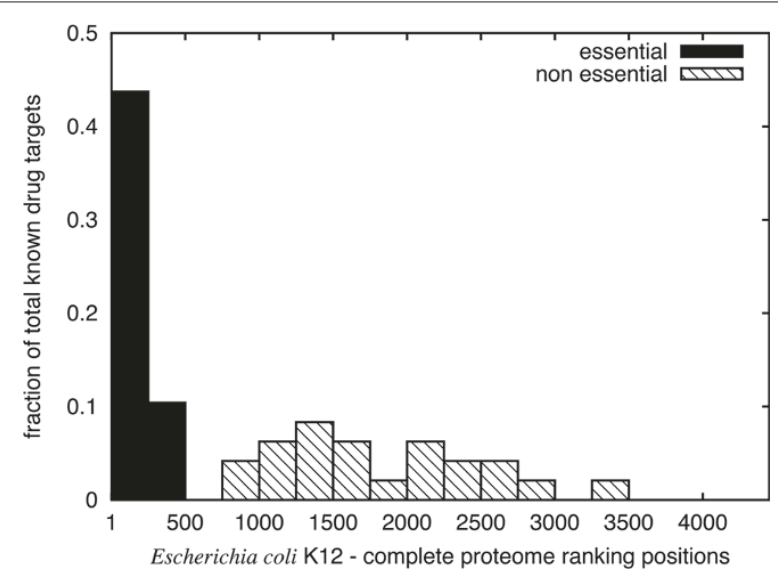

Figure 4 Ranks distribution for known antibacterial targets. The histogram displays the distribution of ranks for 48 DrugBank known targets in Escherichia coli strain K12 within the complete proteome ranking using default parameters. Target proteins known to be essential according to DEG appear at higher ranking positions (solid black), while non-essential proteins appear further down in the ranking (hatch pattern).
Because the function that would unequivocally assign target scores to proteins is highly complex and full of unknowns, the pipeline presented here has been developed with the sole aim to assist the selection of prospective candidates, it is not meant to provide a final or complete list of antibacterial targets. Very often, it will not be used as a ranking tool but to retrieve targetrelevant information for a specific protein and evaluate its pros and cons with respect to other potential candidates. The versatility of the tool, with dynamic features such as the assignation of relative weights at the user's criterion, and the availability of original information, such as predicted, functionally relevant ligand-binding sites, may prove valuable arguments for the microbiologist or medicinal chemist researching on new antibacterial targets.

\section{Conclusions}

We have developed a database and web-based tool for the ranking of proteins from a set of user-selected bacterial proteomes according to a series of antibacterial-drugtarget-like properties. Specifically, the tool evaluates (1) the presence of the protein in other Gram-negative species (currently 494) to assess conservation at this level and, by extension, the potential spectrum of a drug targetting this protein, (2) the identification of the protein as essential for bacterial survival or growth, (3) the presence of homologs in the human genome to assess potential toxicity or side effects of a drug targetting this protein and (4) the presence of paralogs or isoforms of the protein in the same proteome, which could facilitate the development of resistance and reduce effectivity. The user may choose the weight of each of these four properties in the ranking, including negative weights (e.g. for homology to human and presence of isoforms or paralogs). When available, the ranked proteins incorporate, as additional information, any found matches to proteins in the DrugBank (i.e. known drug targets) and the Virulence Factors Database (VFDB), as well as a model of the protein's three-dimensional structure and an analysis of active and regulatory sites to assess druggability of the potential target. This is currently the single resource combining sequence-based and structural information for the identification of potential antibacterial-drug targets in multiple genomes.

Currently, the database covers 74 Gram-negative pathogens, including 224 distinct strains. The number of strains is particularly extended for Acinetobacter baumannii, Escherichia coli, Helicobacter pylori, Pseudomonas aeruginosa and Stenotrophomonas maltophilia (82 strains in total), which are found among a group of Gram-negative pathogens distinguished by prevalence in either community or nosocomial infections and incidence of drug-resistant isolates. Additional strains and 
species will be added to the database in subsequent updates.

\section{Methods}

Normalized sequence-similarity score (NS)

We used the BLASTPGP program [23] with default parameters to scan complete proteomes. Since BLASTPGP E-values may vary depending on the size of the queried database, we aligned all matched pairs and calculated their Smith-Waterman similarity score [44]. We ignored alignments with scores lower than 100 , as previously described [45].

Given that the Smith-Waterman similarity score is related to the size of the alignment, we divided the score by the length of the alignment to obtain a normalized sequence-similarity score (NS). The Smith-Waterman algorithm computes an optimal local alignment, meaning that the NS measure of similarity between two proteins is equivalent to the similarity between their most closely related pair of domains or regions.

\section{Essentiality}

Experimental information regarding gene essentiality is available for a few organisms at the database of essential genes (DEG) [20]. If a particular strain was not available at DEG, we mapped query proteins to essential genes by using BLASTPGP. For each annotated essential gene in a related strain, we scanned the proteome of interest and marked the best hit as an essential gene. Only E-values of 1e-10 or better were considered acceptable for this task. At the time of this writing, we were only able to gather large-scale essential gene information for: Acinetobacter baumannii, Escherichia coli, Helicobacter pylori, Pseudomonas aeruginosa and Vibrio cholerae.

\section{Toxicity}

An antibacterial drug acting on protein targets which are similar to human proteins may also bind these causing adverse effects and/or toxicity. We estimated the potential toxicity of each putative target proportional to the largest NS value obtained after pairwise alignment against the whole human proteome.

\section{Isoforms and paralogs}

If a given drug target presents multiple isoforms or paralogs ('variants'), the pathogen may readily develop resistance by functional substitution mechanisms. It is also possible that the drug may bind both the target and its variants, thus decreasing the antibiotic effect. To assess this parameter for each potential drug target, we counted the amount of variants present in the same proteome. We considered as variants of a protein all similar proteins with a NS value equal or larger than 2 .

\section{Evolutionary conservation among Gram-negative organisms}

We defined a score to estimate the evolutionary conservation of potential targets across Gram-negative (GN) organisms as follows:

$$
G N C_{p}=\sum_{i=1}^{i=n-1} \max \left(N S_{p}\right)
$$

Where $G N C_{p}$ is the Gram-negative conservation score for protein $p$, computed by adding the highest NS value $\left(\max \left(N S_{p}\right)\right)$ obtained against each of the different GN species $(i)$ in the data set, with $n$ being the total number of GN species.

\section{Conservation among strains}

We estimated the evolutionary conservation of proteins among different strains of the same species using the following score:

$$
S C_{p}=\frac{\sum_{j=1}^{j=m-1} \max \left(N S_{p}\right)}{m}
$$

where $S C_{p}$ is the strain conservation score for protein $p$, computed by adding the highest NS value $\left(\max \left(N S_{p}\right)\right)$ obtained against each other strain $(j)$ of the selected species in the data set, with $m$ the total number of distinct strains of the particular species.

\section{Scoring function and ranking of potential drug targets}

Each of the different scores is normalized by the largest value obtained across the selected organisms. Normalized values are then multiplied by 100 to obtain percentages, i.e. final scores range between 0 and 100 .

Each independent score has an associated weight, which can be negative or positive. These weighting values can be set by the user. However, default values are provided as follows. A priori negative features of a putative target (i.e. Toxicity and Paralogs) are given a default weight of 1 , while positive features (e.g. Evolutionary conservation, Essentiality) have a corresponding default weight of 1 .

For each protein in the selected data set, normalized scores are multiplied by their respective weights. The final score for each protein is obtained by summing up all weighted scores. Finally, all proteins in the selected data set are ranked according to their final score in terms of drug-target potential.

\section{Comparative-genomics reference data set}

Sequence data on Gram-negative (GN) organisms was gathered for a total of 770 fully sequenced GN proteomes covering 494 distinct species. GN bacteria species were identified at 'http://www.bacterialphylogeny.com/ bacteria.html' and fully sequenced bacterial proteomes at 'http://www.uniprot.org/taxonomy' using the query 
string: 'bacteria AND complete:yes'. A total of 770 bacterial strains were common to both listings. We downloaded sequence data from 'ftp://ftp.expasy.org/ databases/complete_proteomes/fasta/bacteria/'.

\section{Known drug targets and virulence factors}

Each proteome of interest was compared by means of the BLASTPGP program [23], with default parameters, against known drug targets available at DrugBank [14] and virulence factors available at VFDB [15]. Proteins showing a match with a BLASTPGP E-value $<=1$ e-2 display a link to the related hits in the output table.

\section{Three-dimensional homology models}

An automated homology-modeling pipeline was implemented based on the program MODELLER v9.5 [25]. Briefly, for a given protein sequence the system scans a database of structural templates. It then proceeds to generate homology models using the best possible set of diverse templates that display at least $30 \%$ sequence identity. Finally, the best resulting models are selected using a combination of DOPE and GA341 scores [25].

\section{Competing interests}

The authors declare that they have no competing interests.

\section{Authors' contributions}

$X D, I G$ and AP conceived the project. AP performed the computational work. $A P$ and $X D$ wrote the manuscript. All authors read and approved the final manuscript.

\section{Acknowledgements}

This work has been supported by funding under the Seventh Research Framework Programme of the European Union (ref. HEALTH-F3-2009-223101, AntiPathoGN) and the Spanish Ministry for Science and Innovation (BFU2010-17199). AP acknowledges the FPU Scholarship received from the Spanish Government. The authors acknowledge Manuel Alonso Tarajano for the initial compilation of the Gram-negative reference set.

\section{Author details}

${ }^{1}$ Institute of Biotechnology and Biomedicine (IBB), Universitat Autònoma de Barcelona (UAB), 08193 Bellaterra, Spain. ${ }^{2}$ Departament de Genètica i de Microbiologia, UAB, 08193 Bellaterra, Spain. ${ }^{3}$ Catalan Institution for Research and Advanced Studies (ICREA), 08010 Barcelona, Spain.

Received: 30 September 2013 Accepted: 11 January 2014

Published: 17 January 2014

\section{References}

1. Arias CA, Murray BE: Antibiotic-resistant bugs in the 21 st century - a clinical super-challenge. N Eng/ J Med 2009, 360(5):439-443.

2. Baquero F, Alvarez-Ortega C, Martinez JL: Ecology and evolution of antibiotic resistance. Environ Microbiol Rep 2009, 1(6):469-476.

3. Boucher HW, Talbot GH, Bradley JS, Edwards JE, Gilbert D, Rice LB, Scheld M, Spellberg B, Bartlett J: Bad bugs, no drugs: no ESKAPE! An update from the Infectious Diseases Society of America. Clin Infect Dis 2009, 48:1-12.

4. European Academies Science Advisory Council: Tackling antibacterial resistance in Europe. Tech. rep., EASAC 2007.

5. Souli M, Galani I, Giamarellou H: Emergence of extensively drug-resistant and pandrug-resistant Gram-negative bacilli in Europe. Euro Surveill 2008, 13(47):19045.

6. Talbot GH, Bradley J, Edwards JE, Gilbert D, Scheld M, Bartlett JG: Antimicrobial Availability Task Force of the Infectious Diseases
Society of America: Bad bugs need drugs: an update on the development pipeline from the Antimicrobial Availability Task Force of the Infectious Diseases Society of America. Clin Infect Dis 2006, 42(5):657-668.

7. Payne DJ, Gwynn MN, Holmes DJ, Pompliano DL: Drugs for bad bugs: confronting the challenges of antibacterial discovery. Nat Rev Drug Discov 2007, 6:29-40.

8. Tally FP, DeBruin MF: Development of daptomycin for gram-positive infections. J Antimicrob Chemother 2000, 46(4):523-526.

9. van Hal SJ, Paterson DL: New Gram-positive antibiotics: better than vancomycin?. Curr Opin Infect Dis 2011, 24(6):515-520.

10. Bereket W, Hemalatha K, Getenet B, Wondwossen T, Solomon A, Zeynudin A, Kannan S: Update on bacterial nosocomial infections. Eur Rev Med Pharmacol Sci 2012, 16(8):1039-1044.

11. Akova M, Daikos GL, Tzouvelekis L, Carmeli Y: Interventional strategies and current clinical experience with carbapenemase-producing Gram-negative bacteria. Clin Microbiol Infect 2012, 18(5):439-448.

12. Panjkovich $A$, Daura $X$ : Assessing the structural conservation of protein pockets to study functional and allosteric sites: implications for drug discovery. BMC Struct Bio/ 2010, 10:9.

13. Panjkovich $A$, Daura $X$ : Exploiting protein flexibility to predict the location of allosteric sites. BMC Bioinformatics 2012, 13:273.

14. Wishart DS, Knox C, Guo AC, Cheng D, Shrivastava S, Tzur D, Gautam B, Hassanali M: DrugBank: a knowledgebase for drugs, drug actions and drug targets. Nucleic Acids Res 2008, 36(Database issue):D901-D906.

15. Yang J, Chen L, Sun L, Yu J, Jin Q: VFDB 2008 release: an enhanced web-based resource for comparative pathogenomics. Nucleic Acids Res 2008, 36(Database issue):D539-D542.

16. Chanumolu SK, Rout C, Chauhan RS: UniDrug-target: a computational tool to identify unique drug targets in pathogenic bacteria. PLOS One 2012, 7(3):e32833.

17. Brittnacher MJ, Fong C, Hayden HS, Jacobs MA, Radey M, Rohmer L: PGAT: a multistrain analysis resource for microbial genomes. Bioinformatics 2011, 27(17):2429-2430.

18. Shanmugham B, Pan A: Identification and characterization of potential therapeutic candidates in emerging human pathogen Mycobacterium abscessus: a novel hierarchical in silico approach. PLoS One 2013, 8(3):e59126.

19. White TA, Kell DB: Comparative genomic assessment of novel broad-spectrum targets for antibacterial drugs. Comp Funct Genomics 2004, 5(4):304-327.

20. Zhang $C T$, Zhang R: Gene essentiality analysis based on DEG, a database of essential genes. Methods Mol Biol 2008, 416:391-400

21. Sakharkar KR, Sakharkar MK, Chow VTK: A novel genomics approach for the identification of drug targets in pathogens, with special reference to Pseudomonas aeruginosa. In Silico Biol 2004, 4(3):355-360.

22. Pedruzzi I, Rivoire C, Auchincloss AH, Coudert E, Keller G, de Castro E, Baratin D, Cuche BA, Bougueleret L, Poux S, Redaschi N, Xenarios I, Bridge A: UniProt-Consortium: HAMAP in 2013, new developments in the protein family classification and annotation system. Nucleic Acids Res 2013, 41(Database issue):D584-D589.

23. Altschul SF, Madden TL, Schaeffer AA, Zhang J, Zhang Z, Miller W, Lipman DJ: Gapped BLAST and PSI-BLAST: a new generation of protein database search programs. Nucleic Acids Res 1997, 25(17):3389-3402.

24. Laskowski RA, Rullmannn JA, MacArthur MW, Kaptein R, Thornton JM: AQUA and PROCHECK-NMR: programs for checking the quality of protein structures solved by NMR. J Biomol NMR 1996, 8(4):477-486.

25. Eswar N, Eramian D, Webb B, Shen MY, Sali A: Protein structure modeling with MODELLER. Methods Mol Biol 2008, 426:145-159.

26. Mistry J, Bateman A, Finn RD: Predicting active site residue annotations in the Pfam database. BMC Bioinformatics 2007, 8:298.

27. Huang B, Schroeder M: LIGSITEcsc: predicting ligand binding sites using the Connolly surface and degree of conservation. BMC Struct Biol 2006, 6:19.

28. UniProt Consortium: The Universal Protein Resource (UniProt) 2009. Nucleic Acids Res 2009, 37(Database issue):D169-D174.

29. Fournier PE, Vallenet D, Barbe V, Audic S, Ogata H, Poirel L, Richet H, Robert C, Mangenot S, Abergel C, Nordmann P, Weissenbach J, Raoult D, Claverie JM: Comparative genomics of multidrug resistance in Acinetobacter baumannii. PLoS Genet 2006, 2:e7. 
30. Lee DS, Burd H, Liu J, Almaas E, Wiest O, Barabási AL, Oltvai ZN, Kapatral V: Comparative genome-scale metabolic reconstruction and flux balance analysis of multiple Staphylococcus aureus genomes identify novel anti-microbial drug targets. J Bacterio/ 2009, 191(12):4015-4024.

31. Mills SD: When will the genomics investment pay off for antibacterial discovery? Biochem Pharmacol 2006, 71(7):1096-1102.

32. Coates ARM, Hu Y: Novel approaches to developing new antibiotics for bacterial infections. Br J Pharmacol 2007, 152(8):1147-1154.

33. Kos VN, Desjardins CA, Griggs A, Cerqueira G, Tonder AV, Holden MTG, Godfrey P, Palmer KL, Bodi K, Mongodin EF, Wortman J, Feldgarden M, Lawley T, Gill SR, Haas BJ, Birren B, Gilmore MS: Comparative genomics of vancomycin-resistant Staphylococcus aureus strains and their positions within the clade most commonly associated with Methicillin-resistant S. aureus hospital-acquired infection in the United States. MBio 2012, 3(3):e00112-12.

34. Piras C, Soggiu A, Bonizzi L, Gaviraghi A, Deriu F, Martino LD, lovane G, Amoresano A, Roncada P: Comparative proteomics to evaluate multi drug resistance in Escherichia coli. Mol Biosyst 2012, 8(4):1060-1067.

35. Imming $P$, Sinning $C$, Meyer $A$ : Drugs, their targets and the nature and number of drug targets. Nat Rev Drug Discov 2006, 5(10):821-834.

36. Lee JK, Lee YS, Park YK, Kim BS: Mutations in the gyrA and parC genes in ciprofloxacin-resistant clinical isolates of Acinetobacter baumannii in Korea. Microbiol Immunol 2005, 49(7):647-653.

37. Bax BD, Chan PF, Eggleston DS, Fosberry A, Gentry DR, Gorrec F, Giordano I, Hann MM, Hennessy A, Hibbs M, Huang J, Jones E, Jones J, Brown KK, Lewis CJ, May EW, Saunders MR, Singh O, Spitzfaden CE, Shen C, Shillings A, Theobald AJ, Wohlkonig A, Pearson ND, Gwynn MN: Type IIA topoisomerase inhibition by a new class of antibacterial agents. Nature 2010, 466(7309):935-940.

38. Guilloteau JP, Mathieu M, Giglione C, Blanc V, Dupuy A, Chevrier M, Gil P, Famechon A, Meinnel T, Mikol V: The crystal structures of four peptide deformylases bound to the antibiotic actinonin reveal two distinct types: a platform for the structure-based design of antibacterial agents. J Mol Biol 2002, 320(5):951-962.

39. Khandekar SS, Daines RA, Lonsdale JT: Bacterial beta-ketoacyl-acyl carrier protein synthases as targets for antibacterial agents. Curr Protein Pept Sci 2003, 4:21-29.

40. Nie Z, Perretta C, Lu J, Su Y, Margosiak S, Gajiwala KS, Cortez J, Nikulin V, Yager KM, Appelt K, Chu S: Structure-based design, synthesis, and study of potent inhibitors of beta-ketoacyl-acyl carrier protein synthase III as potential antimicrobial agents. J Med Chem 2005, 48(5):1596-1609.

41. Zhang HJ, Li ZL, Zhu HL: Advances in the research of beta-ketoacyl-ACP synthase III (FabH) inhibitors. Curr Med Chem 2012, 19(8):1225-1237.

42. Juhas M, Stark M, von Mering, C, Lumjiaktase P, Crook DW, Valvano MA Eberl L: High confidence prediction of essential genes in Burkholderia cenocepacia. PLOS One 2012, 7(6):e40064.

43. Umland TC, Schultz LW, MacDonald U, Beanan JM, Olson R, Russo TA: In vivo-validated essential genes identified in Acinetobacter baumannii by using human ascites overlap poorly with essential genes detected on laboratory media. MBio 2012, 3(4):e00113-12.

44. Smith TF, Waterman MS: Identification of common molecular subsequences. J Mol Biol 1981, 147:195-197.

45. Aoki KF, Kanehisa M: Using the KEGG database resource. Curr Protoc Bioinformatics 2005, Chapter 1:Unit 1.12.

doi:10.1186/1471-2164-15-36

Cite this article as: Panjkovich et al:: antibacTR: dynamic antibacterial-drugtarget ranking integrating comparative genomics, structural analysis and experimental annotation. BMC Genomics 2014 15:36.

Submit your next manuscript to BioMed Central and take full advantage of:

- Convenient online submission

- Thorough peer review

- No space constraints or color figure charges

- Immediate publication on acceptance

- Inclusion in PubMed, CAS, Scopus and Google Scholar

- Research which is freely available for redistribution

Submit your manuscript at www.biomedcentral.com/submit
C Biomed Centra 